\title{
Peretinoin after curative therapy of hepatitis C-related hepatocellular carcinoma: a randomized double-blind placebo-controlled study
}

\author{
Kiwamu Okita - Namiki Izumi - Osamu Matsui - Katsuaki Tanaka - Shuichi Kaneko • \\ Hisataka Moriwaki $\cdot$ Kenji Ikeda • Yukio Osaki $\cdot$ Kazushi Numata Kohei Nakachi • \\ Norihiro Kokudo Kazuho Imanaka - Shuhei Nishiguchi · Takuji Okusaka • Yoichi Nishigaki • \\ Susumu Shiomi • Masatoshi Kudo • Kenichi Ido • Yoshiyasu Karino • Norio Hayashi • \\ Yasuo Ohashi · Masatoshi Makuuchi · Hiromitsu Kumada · Peretinoin Study Group
}

Received: 8 November 2013/Accepted: 19 March 2014/Published online: 13 April 2014

(c) The Author(s) 2014. This article is published with open access at Springerlink.com

\begin{abstract}
Background Effective prophylactic therapies have not been established for hepatocellular carcinoma recurrence. Peretinoin represents one novel option for patients with hepatitis $\mathrm{C}$ virus-related hepatocellular carcinoma (HCV$\mathrm{HCC}$ ), and it was tested in a multicenter, randomized, double-blind, placebo-controlled study.

Methods Patients with curative therapy were assigned to one of the following regimens: peretinoin $600,300 \mathrm{mg} /$ day, or placebo for up to 96 weeks. The primary outcome was recurrence-free survival (RFS).
\end{abstract}

This study was presented in part at the 46th annual meeting of the American Society of Clinical Oncology, Chicago, IL, June 4-8, 2010, the annual conference of the International Liver Cancer Association, Montreal, September 10-12, 2010, and the 2011 American Society of Clinical Oncology Gastrointestinal Cancers Symposium, San Francisco, CA, January 20-22, 2011.

Electronic supplementary material The online version of this article (doi:10.1007/s00535-014-0956-9) contains supplementary material, which is available to authorized users.

\section{K. Okita $(\square)$}

Shimonoseki Kohsei Hospital, Kamishinchi-Machi 3-3-8,

Shimonoseki, Yamaguchi 750-0061, Japan

e-mail: k.okita@kousei-h.jp

N. Izumi

Musashino Red Cross Hospital, Sakai-minami-cho 1-26-1,

Musashino, Tokyo 180-8610, Japan

O. Matsui · S. Kaneko

Kanazawa University Hospital, Takara-machi 13-1, Kanazawa,

Ishikawa 920-8641, Japan

K. Tanaka $\cdot$ K. Numata

Yokohama City University Medical Center, Urafune 4-57,

Minami-ku, Yokohama 232-0024, Japan
Results Of the 401 patients initially enrolled, 377 patients were analyzed for efficacy. The RFS rates in the 600-mg group, the 300-mg group, and the placebo group were 71.9, 63.6, and $66.0 \%$ at 1 year, and 43.7, 24.9, and $29.3 \%$ at 3 years, respectively. The primary comparison of peretinoin (300 and 600-mg) with placebo was not significant $(P=0.434)$. The dose-response relationship based on the hypothesis that "efficacy begins to increase at $600 \mathrm{mg} /$ day" was significant $(P=0.023$, multiplicity-adjusted $P=0.048)$. The hazard ratios for RFS in the 600-mg group vs. the placebo group were 0.73 [95\% confidence interval (CI) $0.51-1.03$ ] for the entire study period and 0.27 (95\% CI 0.07-0.96) after 2 years of the randomization. Common adverse events included ascites, increased blood pressure, headache, presence of urine albumin, and increased transaminases.

Conclusions Although the superiority of peretinoin to placebo could not be validated, $600 \mathrm{mg} /$ day was shown to be the optimal dose, and treatment may possibly reduce the recurrence of $\mathrm{HCV}-\mathrm{HCC}$, particularly after 2 years. The

H. Moriwaki

Gifu University Hospital, Yanagido 1-1, Gifu 501-1194, Japan

K. Ikeda $\cdot$ H. Kumada

Toranomon Hospital, Toranomon 2-2-2, Minato-ku,

Tokyo 105-8470, Japan

Y. Osaki

Osaka Red Cross Hospital, Fudegasaki-cho 5-30, Tennoji-ku,

Osaka 543-8555, Japan

K. Nakachi

National Cancer Center Hospital East, Kashiwanoha 6-5-1,

Kashiwa, Chiba 277-8577, Japan 
efficacy and safety of peretinoin $600 \mathrm{mg} /$ day should continue to be evaluated in further studies.

Keywords Liver neoplasms - Recurrence $\cdot$ Multicentric de novo carcinogenesis · Retinoids $\cdot$ Dose-response relationship

\section{Abbreviation \\ CI Confidence interval \\ CT Computed tomography \\ HCC Hepatocellular carcinoma \\ HR Hazard ratio \\ RFS Recurrence-free survival}

\section{Introduction}

Hepatocellular carcinoma (HCC) is the sixth most common cancer in the world, affecting 740,000 people annually [1]. The incidence of HCC has been rising due to an increase in hepatitis-C virus infections [2-4]. Curative resection or ablation is indicated for early HCC [5-7]. However, the 3-year recurrence rate after curative treatment in the general population is $50 \%[8,9]$. Furthermore, the recurrence rate is $>70 \%$ in hepatitis $\mathrm{C}$ virus-positive patients [10]. Recurrence within 2 years of curative treatment is mainly associated with intrahepatic metastasis, whereas multicentric de novo carcinogenesis (second primary HCC) is the common cause of recurrence after 2 years [11, 12]. Most importantly, no effective approach has been established to prevent $\mathrm{HCC}$ recurrence [13-15].

\section{N. Kokudo}

The University of Tokyo Hospital, Hongo 7-3-1, Bunkyo-ku, Tokyo 113-8655, Japan

\section{K. Imanaka}

Osaka Medical Center for Cancer and Cardiovascular Diseases, Nakamichi 1-3-3 Higashinari-ku, Osaka 537-8511, Japan

\section{S. Nishiguchi}

Hyogo College of Medicine Hospital, Mukogawa-cho 1-1, Nishinomiya, Hyogo 663-8501, Japan

T. Okusaka

National Cancer Center Hospital, Tsukiji 5-1-1, Chuo-ku, Tokyo 104-0045, Japan

\section{Y. Nishigaki}

Gifu Municipal Hospital, 7-1 Kashima-cho, Gifu 500-8513, Japan

\section{S. Shiomi}

Osaka City University Hospital, Asahi-machi 1-5-7, Abeno-ku, Osaka 545-8585, Japan
The concept of chemoprevention using retinoids has been proposed as a means to delay or prevent recurrence after treatment of HCC [16]. Peretinoin [ $(2 E, 4 E, 6 E, 10 E)$ 3,7,11,15-Tetramethylhexadeca-2,4,6,10,14-pentaenoic acid] is a synthetic retinoid with a retinoic acid receptor and retinoid $\mathrm{X}$ receptor agonist activity [17]. Peretinoin is known to suppress tumor growth in the human liver by inducing apoptosis and differentiation of liver cancer cells $[18,19]$; it also acts by increasing $\mathrm{p} 21$ protein levels and reducing cyclin D1 levels to inhibit proliferation of these cells [20]. Recently, a small-scale, randomized study has demonstrated that peretinoin $(600 \mathrm{mg} /$ day $)$ reduced HCC recurrence and increased the survival of patients treated with curative therapy $[21,22]$.

Multiple other studies have evaluated the safety of peretinoin dosages. A phase I study evaluated the proportionality of blood concentration using three peretinoin doses (300, 600, and $900 \mathrm{mg} / \mathrm{day})$ [23]. Based on nonclinical studies and phase I studies, a dose of $300 \mathrm{mg} /$ day was assumed to result in a drug concentration in the liver sufficient to produce medicinal action (apoptosis and induction). The $900 \mathrm{mg} /$ day dose resulted in unacceptable hypertension.

Based on these findings and the hypothesis that peretinoin efficacy saturates at $300 \mathrm{mg} /$ day, this clinical study evaluated the efficacy and dose-response relationship of peretinoin in a randomized, double-blind, placebo-controlled study.

In Japan, approximately $70 \%$ of HCC patients are $\mathrm{HCV}$-positive, and there is a higher risk of $\mathrm{HCC}$ recurrence due to $\mathrm{HCV}$ than due to other causes of recurrence. To verify an inhibitory effect on recurrence in a population that is uniform in having a high risk of recurrence, this

M. Kudo

Kinki University Hospital, Ono-higashi 337-2, Sayama, Osaka 589-8511, Japan

K. Ido

Jichi Medical University Hospital, Yakushiji 3311-1, Shimotsuke, Tochigi 329-0498, Japan

Y. Karino

Sapporo Kosei General Hospital, Kita 3-jo Higashi 8-5, Chuo-ku, Sapporo, Hokkaido 060-0033, Japan

N. Hayashi

Kansai Rosai Hospital, Inabaso 3-1-69, Amagasaki, Hyogo 660-8511, Japan

Y. Ohashi

The University of Tokyo, Hongo 7-3-1, Bunkyo-ku, Tokyo 113-8654, Japan

M. Makuuchi

Japanese Red Cross Medical Center, 4-1-22 Hiroo, Shibuya-ku, Tokyo 150-8935, Japan 
study was conducted in $\mathrm{HCV}$-positive patients after having a complete response to treatment.

\section{Methods}

Patients

Patients were recruited from outpatient groups at 41 institutions in Japan. Patients with primary HCV-HCC or first recurrence successfully treated with resection or radiofrequency ablation were included in the study. HCC was diagnosed based on the finding of a typical vascular pattern (hypervascularity in the arterial phase and wash-out in the portal equilibrium phase) on dynamic CT, according to the Consensus-Based Clinical Practice Manual proposed by the Japan Society of Hepatology [5]. Complete response was defined similarly to that of the modified Response Evaluation Criteria in Solid Tumors (modified RECIST) definition and required a diagnosis of a complete cure. Three independent radiologists reviewed all CT images to confirm complete cure. Eligibility criteria included: positive for serum hepatitis $\mathrm{C}$ virus RNA; Child-Pugh liver function class A or B (Table S1); platelet count $\geq 50000 / \mu \mathrm{L}$; and age $\geq 20$ years. Exclusion criteria included: positive for hepatitis B surface antigen; portal invasion with HCC; concurrent use of transcatheter arterial embolization/ chemoembolization for curative treatment; use of other investigational drugs, antitumor drugs, interferon, or vitamin $\mathrm{K} 2$; uncontrollable blood pressure under drug therapy (systolic blood pressure $\geq 160 \mathrm{mmHg}$ or diastolic blood pressure $\geq 100 \mathrm{mmHg}$ ); serious complications; allergy to retinoids or contrast agents for CT; past total gastric resection; and being pregnant or breastfeeding. This study was approved by the institutional review board at each center and conducted in accordance with Good Clinical Practice guidelines and the Declaration of Helsinki. All patients provided written informed consent. The study protocol is registered at JAPIC Clinical Trials: JapicCTI060250 (http://www.clinicaltrials.jp/user/cteSearch.jsp).

\section{Study design}

This study was a multicenter, parallel-group, doubleblind, randomized, placebo-controlled study (Fig. S1). Patients who satisfied the eligibility criteria were assigned to receive peretinoin $600 \mathrm{mg} /$ day, peretinoin $300 \mathrm{mg} / \mathrm{day}$, or placebo at a 1:1:1 ratio. The randomization was centralized, and assignment to study groups was conducted by computer using the minimization method with adjustment for primary tumor/first recurrence and curative treatment (resection/ablation). Equal intra-institutional distribution was ensured. Patients orally ingested the assigned study drug twice daily for up to 96 weeks. Follow-up of individual patients was to be discontinued and study completion would occur when the number of events (HCC recurrence or death) reached 180-200. Patients visited the institutions once every 4 weeks during the study treatment period and once every 12 weeks thereafter. Treatment compliance was evaluated by pill counts. Use of antitumor drugs, interferon, vitamin $\mathrm{K} 2$, vitamin $\mathrm{A}$, or an antiviral drug ribavirin was prohibited during the study duration. All those involved in this study, including patients, were blinded to the treatment regimen and a placebo identical in external appearance to the study drug was used.

\section{Endpoints}

The primary endpoint was recurrence-free survival (RFS), defined as the time from randomization to $\mathrm{HCC}$ recurrence or death from any cause, whichever occurred first. Abdominal dynamic CT was performed every 12 weeks. Tumor markers ( $\alpha$-fetoprotein, Lens culinaris agglutininreactive $\alpha$-fetoprotein isoform, and protein induced by vitamin $\mathrm{K}$ absence or antagonist-II) were measured every 12 weeks. If tumor markers rapidly increased, abdominal dynamic CT was additionally performed. Recurrence of HCC was confirmed based on findings of hypervascularity (nodules enhanced in the arterial phase and washout in the late phase) by dynamic CT images. Recurrence of HCC was judged by three independent radiologists, who reviewed all dynamic CT images. The secondary endpoint was disease-free survival, which was defined as the time from randomization to $\mathrm{HCC}$ recurrence, death from any cause, or onset of secondary tumor, whichever occurred first. For safety assessments, incidences of adverse events were evaluated based on periodic examinations and tests. Laboratory tests were performed collectively at the central laboratory.

\section{Statistical analysis}

The primary comparison of this study was to examine the superiority of peretinoin (300 and 600-mg) to placebo by RFS. RFS was estimated and presented as survival curves for the treatment groups using the Kaplan-Meier method. Subsequently, the dose-response relationship of peretinoin was evaluated. A stratified log-rank test was performed according to the type of curative treatment (resection/ ablation) in the pre-specified three sets of contrasts (Fig. S2). The contrast reflecting the hypothesis that the efficacy of peretinoin saturates at $300 \mathrm{mg} /$ day was tested as the "primary comparison". To evaluate the dose-response relationship of peretinoin, tests were also performed for the two other sets of contrasts ("efficacy increases linearly" 


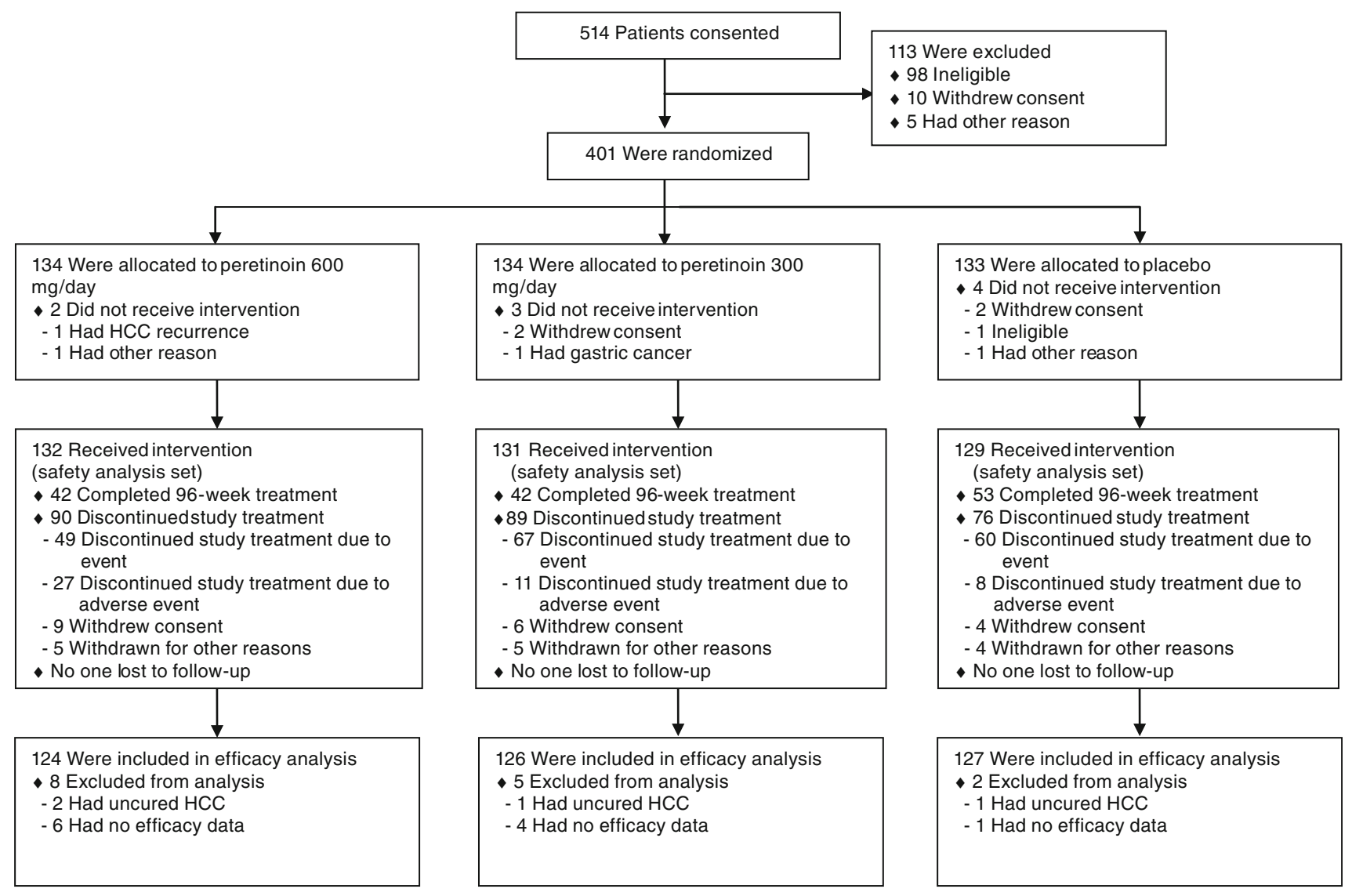

Fig. 1 Flow diagram of study patients

and "efficacy begins to increase at $600 \mathrm{mg} /$ day"). The multiplicity of the three sets of contrasts was adjusted using a permutation test [24].

Sample size was determined based on the primary aim. The number of events required when the clinically useful hazard ratio (HR) supporting a reduction in recurrence was around 0.60 with a 0.025 , one-sided significance level, a 0.90 power, and a $2: 1$ patient ratio [peretinoin (300 and $600-\mathrm{mg}$ ) vs. placebo] was calculated using Freedman's formula [25]. Based on 180 expected events and the 3-year mean duration of follow-up, 120 patients in each treatment group (360 patients in total) were required.

Hazard ratios and $95 \%$ confidence intervals (CIs) for RFS in the peretinoin $600-\mathrm{mg}$ or the $300-\mathrm{mg}$ group vs. placebo group were calculated for the entire study period and at predefined intervals (within 1 year of randomization, at 1-2 years, and after 2 years) with Cox regression analyses using curative treatment as a covariate.

In exploratory, post-hoc, subgroup analysis, the Cox proportional-hazard model was used to evaluate the interaction between baseline characteristics and the effect of peretinoin (peretinoin 600-mg vs. placebo). Factors chosen as baseline characteristics were sex, age, HCC (primary or first recurrence), treatment (local ablation or surgical resection), number of tumor masses, tumor size, and Child-Pugh class.

Efficacy analysis was performed after excluding ineligible patients, patients whose efficacy data were missing, and patients who had never taken the study drug based on the intention-to-treat principle. A two-sided significance level of 0.05 was used. The analysis of disease-free survival was performed in the same manner as the RFS analysis. All patients who had taken the study drug at least once were included in the safety analysis set. Adverse events were classified according to Medical Dictionary for Regulatory Activities (MedDRA) Version 12.0. The dose-response relationship for safety was evaluated using the Cochran-Armitage test.

The independent data and safety monitoring committee performed an interim analysis twice in accordance with the study protocol as follows: first, safety was analyzed when approximately 60 patients had been in the study for at least 1 year; second, safety and efficacy were analyzed when the number of events reached approximately 100. The study was continued after these two interim analyses because none of the discontinuation criteria defined in the study protocol were applicable. 
Table 1 Baseline characteristics of study patients

\begin{tabular}{|c|c|c|c|}
\hline Variable & $\begin{array}{l}\text { Peretinoin } \\
600 \mathrm{mg} / \text { day } n=124 \\
\text { Number (\%) }\end{array}$ & $300 \mathrm{mg} /$ day $n=126$ & Placebo $n=127$ \\
\hline \multicolumn{4}{|l|}{ Gender } \\
\hline Male & $81(65.3)$ & $73(57.9)$ & $87(68.5)$ \\
\hline Female & $43(34.7)$ & $53(42.1)$ & $40(31.5)$ \\
\hline \multicolumn{4}{|l|}{ Age (year) } \\
\hline$<65$ & $37(29.8)$ & $38(30.2)$ & $40(31.5)$ \\
\hline $65-75$ & $63(50.8)$ & $61(48.4)$ & $57(44.9)$ \\
\hline$\geq 75$ & $24(19.4)$ & $27(21.4)$ & $30(23.6)$ \\
\hline Mean (SD) & $68.1(7.1)$ & $68.2(7.7)$ & $68.6(7.8)$ \\
\hline \multicolumn{4}{|l|}{ BMI $\left(\mathrm{kg} / \mathrm{m}^{2}\right)$} \\
\hline$<25$ & $95(76.6)$ & $98(78.4)$ & $106(83.5)$ \\
\hline$\geq 25$ & $29(23.4)$ & 27 (21.6) & $21(16.5)$ \\
\hline \multicolumn{4}{|l|}{ Platelet $\left(\times 10^{4} / \mu \mathrm{L}\right)$} \\
\hline$<10$ & $44(35.5)$ & $49(38.9)$ & $58(45.7)$ \\
\hline$\geq 10$ & $80(64.5)$ & $77(61.1)$ & $69(54.3)$ \\
\hline Means (SD) & $12.0(5.1)$ & $11.5(4.0)$ & $11.4(4.3)$ \\
\hline \multicolumn{4}{|l|}{ Child-Pugh class ${ }^{\mathrm{a}}$} \\
\hline A & $100(80.6)$ & $104(82.5)$ & $106(83.5)$ \\
\hline B & $24(19.4)$ & $22(17.5)$ & $21(16.5)$ \\
\hline \multicolumn{4}{|l|}{$\mathrm{HCC}$} \\
\hline Primary & $111(89.5)$ & $111(88.1)$ & $115(90.6)$ \\
\hline First recurrence & $13(10.5)$ & 15 (11.9) & $12(9.4)$ \\
\hline \multicolumn{4}{|l|}{ Treatment } \\
\hline Local ablation & $78(62.9)$ & $81(64.3)$ & $83(65.4)$ \\
\hline Surgical resection & $46(37.1)$ & $45(35.7)$ & $44(34.6)$ \\
\hline \multicolumn{4}{|c|}{ Number of tumor masses } \\
\hline 1 & $104(83.9)$ & $106(84.1)$ & $105(82.7)$ \\
\hline $2-3$ & $19(15.3)$ & $20(15.9)$ & $21(16.5)$ \\
\hline$\geq 4$ & $1(0.8)$ & $0(0.0)$ & $1(0.8)$ \\
\hline \multicolumn{4}{|l|}{ Tumor size $(\mathrm{cm})$} \\
\hline$<2$ & $56(45.2)$ & $55(43.7)$ & $57(44.9)$ \\
\hline$\geq 2$ & $68(54.8)$ & $71(56.3)$ & $70(55.1)$ \\
\hline \multicolumn{4}{|l|}{$\mathrm{AFP}(\mathrm{ng} / \mathrm{mL})$} \\
\hline$\leq 10$ & $57(46.0)$ & $47(37.3)$ & $45(35.4)$ \\
\hline$>10$ & $67(54.0)$ & $79(62.7)$ & $82(64.6)$ \\
\hline Means (SD) & $44.8(152.8)$ & $37.3(76.3)$ & $39.4(82.4)$ \\
\hline \multicolumn{4}{|l|}{ AFP-L ${ }_{3}(\%)$} \\
\hline$\leq 10$ & $113(91.1)$ & $116(92.1)$ & $117(92.1)$ \\
\hline$>10$ & $11(8.9)$ & $10(7.9)$ & $10(7.9)$ \\
\hline Means (SD) & $3.9(10.1)$ & $3.2(4.8)$ & $4.3(8.3)$ \\
\hline \multicolumn{4}{|l|}{ PIVKA-II (mAU/mL) } \\
\hline$\leq 40$ & $115(92.7)$ & $118(93.7)$ & $118(92.9)$ \\
\hline$>40$ & $9(7.3)$ & $8(6.3)$ & $9(7.1)$ \\
\hline Means (SD) & $42.9(227.0)$ & $26.8(44.1)$ & $86.5(711.3)$ \\
\hline
\end{tabular}

$B M I$ body mass index, HCC hepatocellular carcinoma, AFP alpha-fetoprotein, AFP-L3 alpha-fetoprotein L3, PIVKA-II protein induced by vitamin $\mathrm{K}$ absence or antagonist-II, $S D$ standard deviation

${ }^{a}$ Severity of hepatic dysfunction evaluated on the scale of Child-Pugh class A to C. The analysis excluded patients with class $\mathrm{C}$ hepatic dysfunction 


\section{Results}

\section{Patients}

Patients were recruited from March 14, 2005, through July 30, 2007. The study was terminated on August 27, 2009, because the target number of events (180-200) was achieved. The median follow-up period was 911 days (95\% CI 845-937 days). A total of 401 patients were randomized (Fig. 1). Patient characteristics were comparable among the three treatment groups (Table 1). Patients aged $\geq 65$ years accounted for about $70 \%$ of the study population, and those who were Child-Pugh class A accounted for about $80 \%$ of patients in each treatment group. The mean duration of the study treatment was 416 days (95\% CI 392-441 days) (600-mg group, 398 days; 300-mg group, 410 days; placebo group, 442 days). Of those who were included in the efficacy analysis, 368 patients $(97.6 \%)$ complied with the study treatment for at least $70 \%$ of the time (96.0, 98.4, and $98.4 \%$, respectively).

\section{Efficacy}

A total of 377 patients were analyzed (600-mg group: 124, 300-mg group: 126, placebo group: 127). Figure 2 shows 300, 600-mg Kaplan-Meier curves of the 600-mg group, the 300-mg group, and the placebo group for RFS. In the primary comparison, the effect of peretinoin (300 and 600-mg) on RFS compared to that of the placebo was not significant $(P=0.434)$. As shown in the Kaplan-Meier curves, RFS in the 600-mg group during the entire course of the study trended slightly higher than in the other two groups. The proportions of patients with RFS in the 600-mg group, the 300-mg group, and the placebo group, respectively, were $71.9,63.6$, and $66.0 \%$ in year $1,48.3,43.4$, and $42.3 \%$ in year 2, and 43.7, 24.9, and $29.3 \%$ in Year 3 (Table S2). Hazard ratios for the RFS results of peretinoin $600 \mathrm{mg} /$ day vs. placebo and peretinoin $300 \mathrm{mg} /$ day vs. placebo are shown in Table 2 . The risks were comparable in the 300-mg and placebo groups (HR during the entire study period, 1.06; $95 \%$ CI 0.78-1.45; HR after 2 years, 1.19; $95 \%$ CI 0.55-2.60). In contrast, the risk of recurrence after 2 years of randomization in the 600-mg group decreased by $70 \%$ compared to the placebo group (HR during the entire study period, 0.73; $95 \%$ CI 0.51-1.03; HR after 2 years, 0.27 ; $95 \%$ CI 0.07-0.96). During the study, RFS events were defined as HCC recurrence or death of any cause; there were 53 RFS events observed in patients in the 600-mg group, 80 events in the 300-mg group, and 77 events in the placebo group (Table S2). The analysis of disease-free survival showed comparable results (Fig. S3).
Dose-response relationship

Table 3 shows the results of log-rank tests on the three prespecified comparisons, which were based on the three hypotheses for the dose-response relationship of peretinoin. No significant dose-response relationship was seen in the comparison that tested whether "peretinoin efficacy saturates at $300 \mathrm{mg} /$ day" ( $P=0.434$, the same test applied to the "primary comparison" described previously). When testing whether "peretinoin efficacy increases linearly", no significance was obtained $(P=0.079)$. In contrast, the dose-response relationship was significant in the comparison that tested whether "peretinoin efficacy begins to increase at $600 \mathrm{mg} /$ day" $(P=0.023$, multiplicity-adjusted $P=0.048)$.

\section{Post-hoc subgroup analysis}

The effect of the $600 \mathrm{mg}$ dosage of peretinoin observed in the entire study population was consistent with each subgroup except in patients with tumor size $\geq 2 \mathrm{~cm}$ and ChildPugh B (Fig. 3a). There were significant treatment effect interactions with tumor size and Child-Pugh class ( $P=0.039$ and $P=0.035$, respectively), and the interaction with Child-Pugh class was the largest both in magnitude of the effect size and in statistical significance. Kaplan-Meier curves of the 600-mg group vs. placebo group for RFS in patients with Child-Pugh A are shown in Fig. 3b.

\section{Safety}

A total of 392 patients were analyzed (600-mg group: 132, 300-mg group: 131, placebo group: 129). The overall incidence of adverse events was $95.5 \%(126 / 132)$ in the 600-mg group, $93.9 \%(123 / 131)$ in the 300-mg group, and $90.7 \%(117 / 129)$ in the placebo group. Common adverse events that occurred in $\geq 10 \%$ of patients in either the 600 or 300-mg of the treatment groups included ascites, diarrhoea, oesophageal varices, nasopharyngitis, back pain, headache, oedema peripheral, albumin urine present, increased blood pressure and increased transaminases (Table 4). Incidences of these events mainly increased with peretinoin dose. Most of these events were mild or moderate in severity and controllable. The proportion of adverse events which resulted in discontinuation of the study treatment increased with dose: $15.9 \%$ (21/132) in the 600-mg group, $6.9 \%(9 / 131)$ in the 300-mg group, and $4.7 \%(6 / 129)$ in the placebo group $(P=0.002)$. These events included onychoclasis, headache, anemia, renal impairment, edema, peripheral edema, and increased transaminases. 
The overall incidence of serious adverse events increased with peretinoin dose: $31.1 \%$ (41/132) in the 600-mg group, $29.8 \%(39 / 131)$ in the 300-mg group, and $20.2 \%(26 / 129)$ in the placebo group $(P=0.048)$. Seven patients treated with peretinoin died from serious adverse events. They were all aged $\geq 65$ years (including three aged $\geq 75$ years). The baseline Child-Pugh class was $\mathrm{A}$ in three patients and B in four. The causes of death varied: three patients in the 600-mg group died of cardiorespiratory arrest, sepsis, and hepatic failure, and four in the 300-mg group died of pneumonia, hepatic failure, sudden death, and diffuse large B-cell lymphoma.

\section{Discussion}

This study could not confirm the efficacy of peretinoin (300 and $600 \mathrm{mg} /$ day) for reducing the recurrence of HCV-HCC. Based on the previous Phase I study [26], peretinoin was assumed to be effective from the $300 \mathrm{mg} /$ day dose level; thus, this study was designed to compare peretinoin (300 and 600-mg) dosage groups to a placebo group as the primary objective. Unfortunately, the results did not achieve statistical significance. The number of recurrences and the

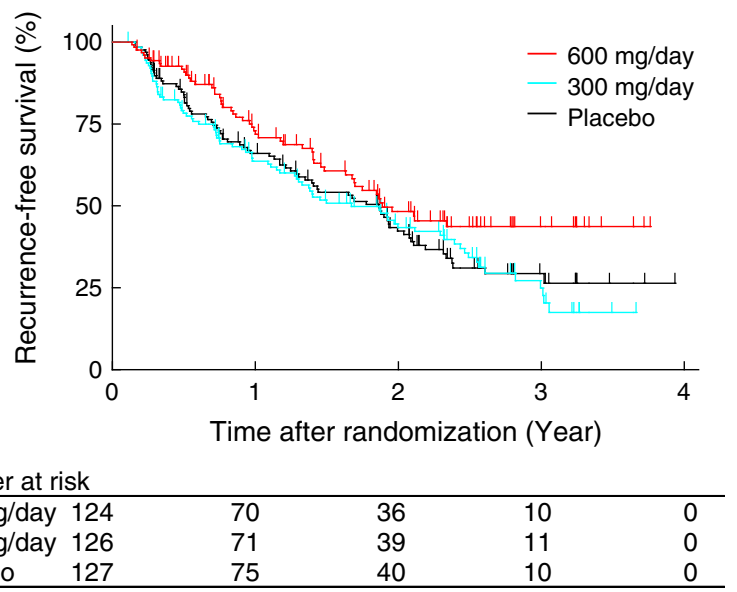

\begin{tabular}{llllll}
\multicolumn{2}{l}{ Number at risk } \\
\hline $600 \mathrm{mg} /$ day & 124 & 70 & 36 & 10 & 0 \\
$300 \mathrm{mg} /$ day & 126 & 71 & 39 & 11 & 0 \\
Placebo & 127 & 75 & 40 & 10 & 0 \\
\hline
\end{tabular}

Fig. 2 Kaplan-Meier curves for recurrence-free survival
HR observed in the peretinoin 300-mg group were equivalent to that of the placebo group, which indicated the dose level of peretinoin $300 \mathrm{mg} /$ day was ineffective in this patient population. Sample size of the study was determined to test the primary comparison of peretinoin (300 and $600-\mathrm{mg}$ ) group vs. placebo group with number of patients in $2: 1$ ratio. Since there was no statistically significant difference, the comparisons between peretinoin 300-mg or $600-\mathrm{mg}$ to the placebo group were underpowered.

The gene expression pattern in the liver before and after peretinoin treatment was examined in a clinical pharmacological study performed in humans simultaneously with this study. Genes expected to result in inhibition of recurrence, including retinoid-induced genes, interferonand tumor suppressor-related genes, and hepatocyte differentiation genes, were among the genes that changed markedly before and after treatment with $600 \mathrm{mg} /$ day. However, there was little change or no change in expression of these genes with treatment at $300 \mathrm{mg} /$ day [27]. Consequently, while it was assumed that non-clinical studies and pharmacokinetic results showed that treatment of $300 \mathrm{mg} /$ day elicited sufficient medicinal action, the above results of treatment with $300 \mathrm{mg} /$ day in humans shows insufficient change in gene expression, and as such, probably does not confirm the previous result.

The optimal dose of peretinoin was found to be $600 \mathrm{mg} /$ day, and it was possibly effective, particularly after 2 years

Table 3 Dose-response relationship of peretinoin for recurrence-free survival

\begin{tabular}{llll}
\hline $\begin{array}{l}\text { Set of contrast [Placebo, } \\
300 \mathrm{mg} / \text { day, } 600 \mathrm{mg} / \text { day }]\end{array}$ & $\begin{array}{l}\text { Standardized } \\
\log ^{2} \text {-rank score }\end{array}$ & $P$-value & $\begin{array}{l}\text { Adjusted } \\
P \text {-value }\end{array}$ \\
\hline $\begin{array}{c}{[-2,1,1] \text { Efficacy saturates }} \\
\text { at } 300 \mathrm{mg} / \text { day }\end{array}$ & -0.782 & 0.434 & - \\
$\begin{array}{c}{[-1,0,1] \text { Efficacy increases }} \\
\text { linearly }\end{array}$ & -1.756 & 0.079 & - \\
$\begin{array}{l}\quad-1,-1,2] \text { Efficacy begins } \\
\text { to increase at } 600 \mathrm{mg} / \text { day }\end{array}$ & -2.269 & 0.023 & $0.048^{\mathrm{b}}$ \\
\hline
\end{tabular}

${ }^{a}$ Stratified log-rank test based on surgical procedure

b Multiplicity between contrasts adjusted by permutation test

Table 2 Hazard ratios for recurrence-free survival

\begin{tabular}{lllll}
\hline & \multicolumn{2}{l}{ Hazard ratio $(95 \% \mathrm{CI})$} & & \\
\cline { 2 - 5 } & $<1$ year & $1-2$ years & $\geq 2$ years & Overall study period \\
\hline Peretinoin $600 \mathrm{mg} /$ day vs. placebo & $0.72(0.45-1.17)$ & $0.93(0.52-1.66)$ & $0.27(0.07-0.96)$ & $0.73(0.51-1.03)$ \\
Peretinoin $300 \mathrm{mg}$ /day vs. placebo & $1.11(0.73-1.70)$ & $0.89(0.50-1.60)$ & $1.19(0.55-2.60)$ & $1.06(0.78-1.45)$ \\
\hline
\end{tabular}

Hazard ratios for the predetermined periods starting on the day of patient registration and for the entire study period, estimated for peretinoin 600 and $300 \mathrm{mg} /$ day against placebo in a Cox regression analysis with surgical procedure as a covariate. Patients with no events and uncensored patients at the start of predetermined period were included; those with an event and censored patients at the end of predetermined period were censored 


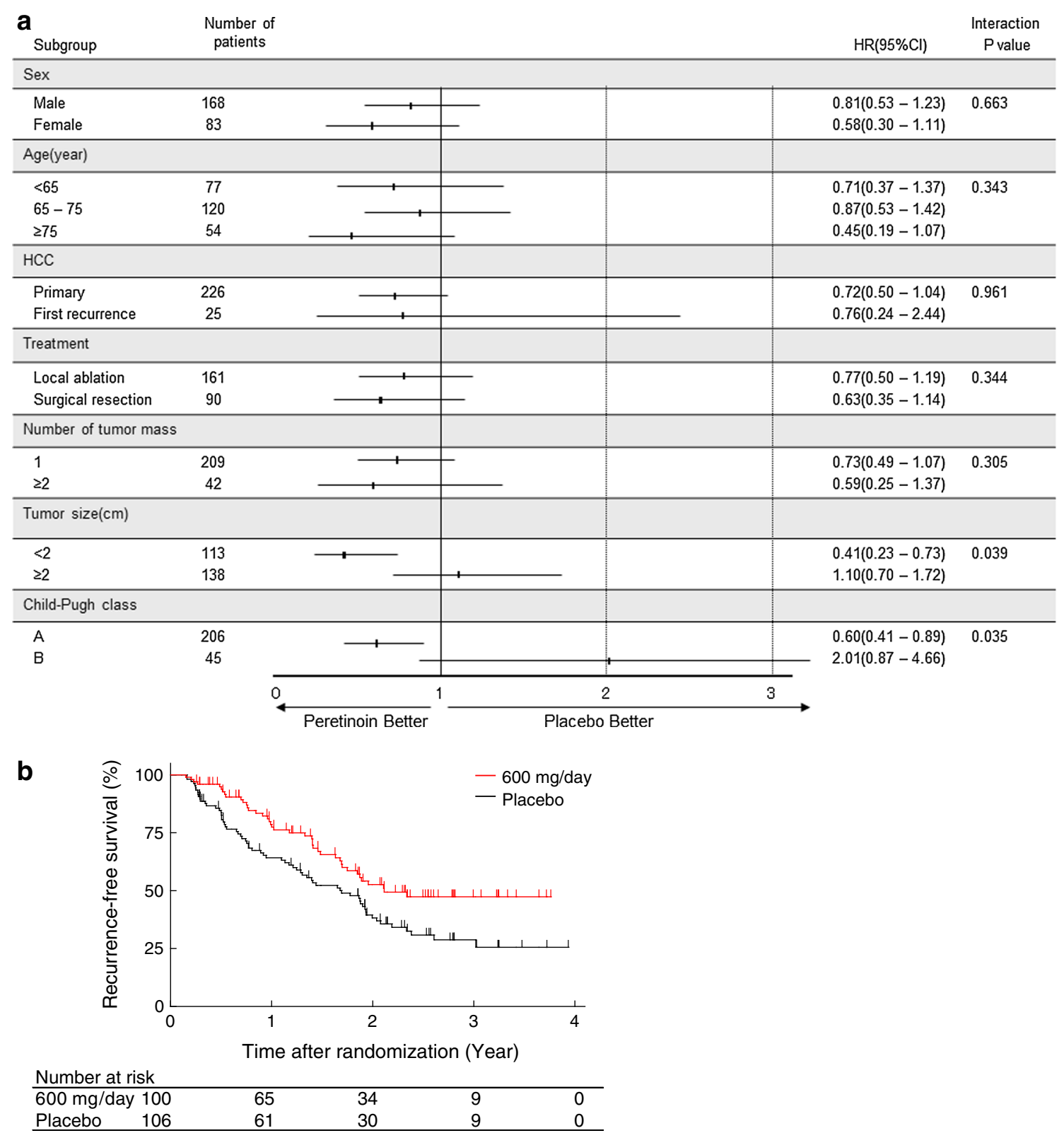

Fig. 3 a Exploratory, post-hoc, subgroup analysis of hazard ratio for recurrence-free survival. b Exploratory, post-hoc, subgroup analysis for recurrence-free survival

from curative treatment. A previous study reported that the overall HR for peretinoin $600 \mathrm{mg} /$ day vs. placebo was 0.31 (95\% CI 0.12-0.78) [21]. This result is comparable to the HR after 2 years $(0.27$; $95 \%$ CI $0.07-0.96)$ in our study. While the previous study evaluated recurrence of only de novo carcinogenesis (second primary HCC), this study evaluated both intrahepatic metastasis and de novo carcinogenesis. However, multicentric de novo carcinogenesis is the common cause of recurrence after 2 years $[11,12]$.

Adverse reactions associated with other retinoids [26] (e.g., mucocutaneous symptoms such as cheilitis and peeling skin, abnormal lipid metabolism, musculoskeletal disorders, and ocular symptoms) occurred infrequently in patients treated with peretinoin. Ascites and increased blood pressure were specific to peretinoin. Blood pressure should be periodically monitored before and during treatment with peretinoin and controlled with antihypertensives as appropriate. Seven patients died from various adverse events. Associations between peretinoin and these adverse events were undeterminable, because all seven patients who died from adverse events were aged $\geq 65$ years, and four among them had Child-Pugh B hepatic impairment. Thus, age and advanced hepatic impairment could have been the causative factor rather than the treatment. The 
Table 4 Adverse events in safety analysis set

\begin{tabular}{|c|c|c|c|c|c|c|}
\hline \multirow[t]{3}{*}{ Adverse events } & \multicolumn{4}{|l|}{ Peretinoin } & \multirow{2}{*}{\multicolumn{2}{|c|}{ Placebo $n=129$}} \\
\hline & \multicolumn{2}{|c|}{$600 \mathrm{mg} /$ day $n=132$} & \multicolumn{2}{|c|}{$300 \mathrm{mg} /$ day $n=131$} & & \\
\hline & $\begin{array}{l}\text { Total } \\
\text { Number }(\%)\end{array}$ & Serious & Total & Serious & Total & Serious \\
\hline Overall incidence & $126(95.5)$ & $41(31.1)$ & $123(93.9)$ & $39(29.8)$ & $117(90.7)$ & $26(20.2)$ \\
\hline \multicolumn{7}{|l|}{ Gastrointestinal disorders } \\
\hline Ascites & $21(15.9)$ & $8(6.1)$ & $15(11.5)$ & $1(0.8)$ & $8(6.2)$ & $1(0.8)$ \\
\hline Diarrhoea & $16(12.1)$ & $1(0.8)$ & $10(7.6)$ & 0 & $7(5.4)$ & 0 \\
\hline Varices oesophageal & $13(9.8)$ & $5(3.8)$ & $15(11.5)$ & $7(5.3)$ & $11(8.5)$ & $4(3.1)$ \\
\hline Constipation & $8(6.1)$ & 0 & $10(7.6)$ & 0 & $5(3.9)$ & 0 \\
\hline Abdominal discomfort & $8(6.1)$ & 0 & $4(3.1)$ & 0 & $2(1.6)$ & 0 \\
\hline Stomatitis & $7(5.3)$ & 0 & $5(3.8)$ & 0 & $2(1.6)$ & 0 \\
\hline Nausea & $7(5.3)$ & 0 & $2(1.5)$ & 0 & $6(4.7)$ & 0 \\
\hline Cheilitis & $7(5.3)$ & 0 & $1(0.8)$ & 0 & $0(0.0)$ & 0 \\
\hline Gastritis & $4(3.0)$ & 0 & $7(5.3)$ & 0 & $2(1.6)$ & 0 \\
\hline Gastric polyps & $1(0.8)$ & 0 & $12(9.2)$ & 0 & $10(7.8)$ & 0 \\
\hline \multicolumn{7}{|l|}{ Infections and infestations } \\
\hline Nasopharyngitis & $50(37.9)$ & 0 & $57(43.5)$ & $1(0.8)$ & $46(35.7)$ & 0 \\
\hline Cystitis & $9(6.8)$ & 0 & $6(4.6)$ & 0 & $4(3.1)$ & 0 \\
\hline Urinary tract infection & $8(6.1)$ & 0 & $6(4.6)$ & $3(2.3)$ & $0(0.0)$ & 0 \\
\hline \multicolumn{7}{|l|}{ Eye disorders } \\
\hline Cataract & $5(3.8)$ & $2(1.5)$ & $7(5.3)$ & $3(2.3)$ & $4(3.1)$ & $2(1.6)$ \\
\hline \multicolumn{7}{|c|}{ Musculoskeletal and connective tissue disorders } \\
\hline Back pain & $17(12.9)$ & 0 & $11(8.4)$ & 0 & $10(7.8)$ & 0 \\
\hline Arthralgia & $7(5.3)$ & 0 & $5(3.8)$ & 0 & $8(6.2)$ & 0 \\
\hline Muscle spasms & $3(2.3)$ & 0 & $7(5.3)$ & 0 & $7(5.4)$ & 0 \\
\hline \multicolumn{7}{|l|}{ Blood and lymphatic system disorders } \\
\hline Anemia & $7(5.3)$ & 0 & $1(0.8)$ & 0 & $2(1.6)$ & 0 \\
\hline \multicolumn{7}{|l|}{ Vascular disorders } \\
\hline Hypertension & $12(9.1)$ & 0 & $10(7.6)$ & 0 & $4(3.1)$ & 0 \\
\hline \multicolumn{7}{|c|}{ Respiratory, thoracic and mediastinal disorders } \\
\hline Cough & $4(3.0)$ & 0 & $7(5.3)$ & 0 & $9(7.0)$ & 0 \\
\hline Upper respiratory tract inflammation & $2(1.5)$ & 0 & $5(3.8)$ & 0 & $7(5.4)$ & 0 \\
\hline \multicolumn{7}{|c|}{ Injury, poisoning and procedural complications } \\
\hline Contusion & $8(6.1)$ & 0 & $7(5.3)$ & 0 & $7(5.4)$ & 0 \\
\hline \multicolumn{7}{|l|}{ Nervous system disorders } \\
\hline Headache & $17(12.9)$ & 0 & $15(11.5)$ & 0 & $11(8.5)$ & 0 \\
\hline Dizziness & $9(6.8)$ & 0 & $5(3.8)$ & 0 & $4(3.1)$ & 0 \\
\hline \multicolumn{7}{|c|}{ General disorders and administration site conditions } \\
\hline Edema peripheral & $16(12.1)$ & 0 & $11(8.4)$ & 0 & $11(8.5)$ & 0 \\
\hline Pyrexia & $12(9.1)$ & $2(1.5)$ & $13(9.9)$ & $1(0.8)$ & $8(6.2)$ & 0 \\
\hline Edema & $10(7.6)$ & 0 & $3(2.3)$ & 0 & $4(3.1)$ & 0 \\
\hline \multicolumn{7}{|l|}{ Metabolism and nutrition disorders } \\
\hline Diabetes mellitus & $3(2.3)$ & $1(0.8)$ & $7(5.3)$ & 0 & $9(7.0)$ & 0 \\
\hline \multicolumn{7}{|l|}{ Skin and subcutaneous tissue disorders } \\
\hline Pruritus & $11(8.3)$ & 0 & $12(9.2)$ & 0 & $9(7.0)$ & 0 \\
\hline Rash & $7(5.3)$ & 0 & $9(6.9)$ & 0 & $9(7.0)$ & 0 \\
\hline Nail disorder & $4(3.0)$ & 0 & $7(5.3)$ & 0 & $2(1.6)$ & 0 \\
\hline
\end{tabular}


Table 4 continued

\begin{tabular}{|c|c|c|c|c|c|c|}
\hline \multirow[t]{3}{*}{ Adverse events } & \multicolumn{4}{|l|}{ Peretinoin } & \multirow{2}{*}{\multicolumn{2}{|c|}{ Placebo $n=129$}} \\
\hline & \multicolumn{2}{|c|}{$600 \mathrm{mg} /$ day $n=132$} & \multicolumn{2}{|c|}{$300 \mathrm{mg} /$ day $n=131$} & & \\
\hline & $\begin{array}{l}\text { Total } \\
\text { Number }(\%)\end{array}$ & Serious & Total & Serious & Total & Serious \\
\hline \multicolumn{7}{|l|}{ Investigations } \\
\hline Albumin urine present & $29(22.0)$ & 0 & $14(10.7)$ & 0 & $8(6.2)$ & 0 \\
\hline Blood pressure increased & $26(19.7)$ & 0 & $20(15.3)$ & 0 & $19(14.7)$ & $1(0.8)$ \\
\hline Transaminases increased & $23(17.4)$ & 0 & $10(7.6)$ & 0 & 15 (11.6) & 0 \\
\hline Protein urine present & $8(6.1)$ & 0 & $2(1.5)$ & 0 & $0(0.0)$ & 0 \\
\hline Blood urine present & $5(3.8)$ & 0 & $7(5.3)$ & 0 & $3(2.3)$ & 0 \\
\hline Gamma-glutamyl transferase increased & $3(2.3)$ & 0 & $5(3.8)$ & 0 & $12(9.3)$ & 0 \\
\hline
\end{tabular}

Adverse events occurred in $\geq 5 \%$ of patients in any of the treatment groups in the safety analysis set, as shown in Medical Dictionary for Regulatory Activities (MedDRA) Version 12.0. Adverse events were classified as being serious or non-serious in accordance with definition adopted by the International Conference on Harmonization

fact that $70 \%$ of study patients were aged $\geq 65$ years attests to the overall tolerability of peretinoin in elderly patients. The Child-Pugh class could also affect peretinoin efficacy. The guidelines for clinical studies of HCC recommend inclusion of patients with Child-Pugh A in clinical trials because death from cirrhosis in patients with Child-Pugh B or C could mask the efficacy of treatment [28]. The proportion of patients with Child-Pugh A was about $80 \%$ in this study. Subgroup analysis revealed that the effect of peretinoin 600-mg in Child-Pugh A patients was significant (HR, 0.60; $95 \%$ CI 0.41-0.89), even though these analyses were post-hoc and exploratory. Recently, a confirmatory peretinoin study was initiated and will be focused on Child-Pugh A patients.

Systemic chemotherapy, immunotherapy, and interferon have not been established as a standard treatment for the prevention of HCV-HCC [13-15]. Through its antiviral action, interferon delays the progression of cirrhosis and hepatic impairment and prolongs survival (pooled risk ratio, 0.65 ; $95 \%$ CI $0.52-0.80$ ). However, it does not significantly reduce recurrence (pooled risk ratio, 0.86; $95 \%$ CI 0.76-0.97) [13]. Peretinoin was previously shown to decrease the risk of recurrence of de novo carcinogenesis by about $70 \%$ [20], and this result was reproduced in this study. Considerably prolonged survival was observed (risk ratio, $0.3 ; 95 \%$ CI 0.1-0.8) [21].

A previous study revealed that $25 \%$ of adverse reactions to interferon were severe, resulting in treatment discontinuation or dose reduction [13]. While safety issues prevent the use of interferon in elderly patients [29, 30], this study indicated that peretinoin was well tolerated by such elderly patients.

One limitation of this study is that the median follow-up period of 2.5 years may be considered short. Since recurrences due to de novo carcinogenesis peak after 4 years postoperatively [11], the reduction of de novo carcinogenesis due to peretinoin may have been more accurately reflected with a longer follow-up period. However, the sample size at 2 years in this study (about 40 patients in each group) was comparable to the sample size of a previous study [21] and adequate to statistically evaluate the efficacy of peretinoin. Future studies should, however, include longer follow-up analysis if feasible.

In this study, the superiority of peretinoin (300 and $600-\mathrm{mg}$ ) to placebo could not be evaluated. Peretinoin $600 \mathrm{mg} /$ day was found to be the optimal dose, and it could possibly reduce the recurrence of HCV-HCC. Although the HR for the RFS in the peretinoin $600 \mathrm{mg} /$ day vs. placebo was not statistically significant for the entire study period, the significant reduction by $>70 \%$ in recurrence after 2 years is clinically meaningful and consistent with previous study results [21]. Further confirmatory studies on Child-Pugh class A patients are worth conducting and would continue to ensure the efficacy of the peretinoin $600 \mathrm{mg} /$ day regimen and thoroughly explore its safety.

Acknowledgments The authors would like to thank Yoshiro Niitsu, Yutaka Aoyagi, Kunihiko Hayasi, Shigehiro Katayama, and Akira Itabashi, who all served on the independent data and safety monitoring committee; special thanks also goes to Tomoaki Ichikawa and Kazuhiko Ueda who served as independent CT image reviewers. The authors would like to acknowledge Statcom Co., Ltd, for their assistance in the preparation and editing of this manuscript. This study was financially supported by Kowa Company, Ltd. Additionally, the authors thank the following principal investigators who enrolled patients in the peretinoin study: (The principal investigators listed below are members of the Peretinoin Study Group) Toshiya Kamiyama, Yoshiyasu Karino, Kunihiko Tsuji, Kazuhiro Kasai, Kenichi Ido, Norio Isoda, Norihiro Kokudo, Fuminori Moriyasu, Shigeki Arii, Takuji Okusaka, Junji Furuse, Kohei Nakachi, Katsumi Sasaki, Kenji Ikeda, Namiki Izumi, Shigehiro Kokubu, Takahide Nakazawa, 
Kazushi Numata, Hisataka Moriwaki, Yoichi Nishigaki, Fumihiro Urano, Susumu Shiomi, Masatoshi Kudo, Kiyohide Kioka, Yukio Osaki, Michio Kato, Eiji Mita, Hiroshi Kasugai, Kazuho Imanaka, Harumasa Yoshihara, Toshihito Seki, Takefumi Nakamura, Toyokazu Fukunaga, Shuhei Nishiguchi, Kazuaki Chayama, Mikiya Kitamoto, Isao Sakaida, Satoyoshi Yamashita, Norio Horiike, Yoichi Hiasa, Makoto Tokumatsu, Nobuhiko Higashi, Shotaro Sakisaka, Yozo Iida, Hiromi Ishibashi, Takashi Kanematsu, Masataka Seike, Yutaka Sasaki, and Hirohito Tsubouchi.

Conflict of interest Izumi has received lecture fees from MSD, Chugai Pharmaceutical, Daiichi Sankyo, and Bayer. Matsui has received consulting fees from Kowa, a research grant from Bayer, a grant from PSP, and other financial support from Eisai and Bayer. Kaneko has received research grants from Bayer and Nippon Shinyaku, and grants from Chugai Pharmaceutical, MSD, Kowa, Mitsubishi Tanabe Pharma, Bristol-Myers Squibb, Eli Lilly, Boehringer Ingelheim Japan, Kyowa Hakko Kirin, Dainippon Sumitomo Pharma, Takeda Pharmaceutical, Eisai, Daiichi Sankyo, AstraZeneca, Novartis Pharma, Astellas Pharma, Teijin Pharma, Zeria Pharmaceutical, Sanofi, Toray Industries, Shionogi, Novo Nordisk Pharma, and Pfizer Japan. Moriwaki has received grants from Ajinomoto Pharmaceuticals, Chugai Pharmaceutical, MSD, Mitsubishi Tanabe Pharma, Otsuka Pharmaceutical, and Bristol-Myers Squibb, and lecture fees from Otsuka Pharmaceutical and Ajinomoto Pharmaceuticals. Kokudo has received a research grant from Bayer, and grants from Chugai Pharmaceutical and Yakult. Nishiguchi has received grants from Chugai Pharmaceutical, MSD, Ajinomoto Pharmaceuticals, and Otsuka Pharmaceutical. Okusaka has received research grants from Chugai Pharmaceutical, Kowa, Otsuka Pharmaceutical, Yakult, Eli Lilly, Taiho Pharmaceutical, Boehringer Ingelheim Japan, Kyowa Hakko Kirin, Ono Pharmaceutical, Takeda Bio Development Center, and the Shizuoka Industrial Foundation. Shiomi has received a research grant from Nihon Medi-Physics. Kudo has received lecture fees from Bayer, and grants from MSD and Takeda Pharmaceutical. Ido has received consulting fees from Kowa. Hayashi has a received grant from Chugai Pharmaceutical. Ohashi has received lecture fees from Kowa Pharmaceutical, and grants from Kowa Pharmaceutical and Astellas Pharma. Kumada holds a patent on SRL, and has received lecture fees from MSD, Dainippon Sumitomo Pharma, Ajinomoto Pharmaceuticals, Mitsubishi Tanabe Pharma, and Bristol-Myers Squibb. The other authors declare that they have no conflicts of interest.

Open Access This article is distributed under the terms of the Creative Commons Attribution Noncommercial License which permits any noncommercial use, distribution, and reproduction in any medium, provided the original author(s) and the source are credited.

\section{References}

1. Ferlay J, Shin HR, Bray F, et al. Estimates of worldwide burden of cancer in 2008: GLOBOCAN 2008. Int J Cancer. 2010;127:2893-917.

2. Altekruse SF, McGlynn KA, Reichman ME. Hepatocellular carcinoma incidence, mortality, and survival trends in the United States from 1975 to 2005. J Clin Oncol. 2009;27:1485-91.

3. Armstrong GL, Wasley A, Simard EP, et al. The prevalence of hepatitis C virus infection in the United States, 1999 through 2002. Ann Intern Med. 2006;144:705-14.

4. El-Serag HB, Davila JA, Petersen NJ, et al. The continuing increase in the incidence of hepatocellular carcinoma in the United States: an update. Ann Intern Med. 2003;139:817-23.

5. Kudo M, Okanoue T. Japan Society of H. Management of hepatocellular carcinoma in Japan: consensus-based clinical practice manual proposed by the Japan Society of Hepatology. Oncology. 2007;72(Suppl 1):2-15.

6. Llovet JM, Bru C, Bruix J. Prognosis of hepatocellular carcinoma: the BCLC staging classification. Semin Liver Dis. 1999;19:329-38.

7. Llovet JM, Burroughs A, Bruix J. Hepatocellular carcinoma. Lancet. 2003;362:1907-17.

8. Castells A, Bruix J, Bru C, et al. Treatment of small hepatocellular carcinoma in cirrhotic patients: a cohort study comparing surgical resection and percutaneous ethanol injection. Hepatology. 1993;18:1121-6.

9. Llovet JM, Schwartz M, Mazzaferro V. Resection and liver transplantation for hepatocellular carcinoma. Semin Liver Dis. 2005;25:181-200.

10. Shiratori Y, Shiina S, Teratani T, et al. Interferon therapy after tumor ablation improves prognosis in patients with hepatocellular carcinoma associated with hepatitis $\mathrm{C}$ virus. Ann Intern Med. 2003;138:299-306.

11. Imamura H, Matsuyama Y, Tanaka E, et al. Risk factors contributing to early and late phase intrahepatic recurrence of hepatocellular carcinoma after hepatectomy. J Hepatol. 2003;38:200-7.

12. Mazzaferro V, Romito R, Schiavo M, et al. Prevention of hepatocellular carcinoma recurrence with alpha-interferon after liver resection in HCV cirrhosis. Hepatology. 2006;44:1543-54.

13. Breitenstein S, Dimitroulis D, Petrowsky H, et al. Systematic review and meta-analysis of interferon after curative treatment of hepatocellular carcinoma in patients with viral hepatitis. Br J Surg. 2009;96:975-81.

14. Mathurin P, Raynard B, Dharancy S, et al. Meta-analysis: evaluation of adjuvant therapy after curative liver resection for hepatocellular carcinoma. Aliment Pharmacol Ther. 2003;17:1247-61.

15. Samuel M, Chow PK, Chan Shih-Yen E, et al. Neoadjuvant and adjuvant therapy for surgical resection of hepatocellular carcinoma. Cochrane Database Syst Rev. 2009:CD001199.

16. Sporn MB, Newton DL. Chemoprevention of cancer with retinoids. Fed Proc. 1979;38:2528-34.

17. Araki H, Shidoji Y, Yamada Y, et al. Retinoid agonist activities of synthetic geranyl geranoic acid derivatives. Biochem Biophys Res Commun. 1995;209:66-72.

18. Nakamura N, Shidoji Y, Yamada Y, et al. Induction of apoptosis by acyclic retinoid in the human hepatoma-derived cell line, HuH-7. Biochem Biophys Res Commun. 1995;207:382-8.

19. Yasuda I, Shiratori Y, Adachi S, et al. Acyclic retinoid induces partial differentiation, down-regulates telomerase reverse transcriptase mRNA expression and telomerase activity, and induces apoptosis in human hepatoma-derived cell lines. J Hepatol. 2002;36:660-71.

20. Suzui M, Masuda M, Lim JT, et al. Growth inhibition of human hepatoma cells by acyclic retinoid is associated with induction of p21(CIP1) and inhibition of expression of cyclin D1. Cancer Res. 2002;62:3997-4006.

21. Muto Y, Moriwaki H, Ninomiya M, Hepatoma Prevention Study Group, et al. Prevention of second primary tumors by an acyclic retinoid, polyprenoic acid, in patients with hepatocellular carcinoma. N Engl J Med. 1996;334:1561-7.

22. Muto Y, Moriwaki H, Saito A. Prevention of second primary tumors by an acyclic retinoid in patients with hepatocellular carcinoma. N Engl J Med. 1999;340:1046-7.

23. Okusaka T, Ueno H, Ikeda M, et al. Phase I and pharmacokinetic clinical trial of oral administration of the acyclic retinoid NIK333. Hepatol Res. 2011;41:542-52.

24. Westfall P, Young S. Resampling-based multiple testing: examples and methods for $p$-value adjustment. 1st ed. New York: Wiley-Interscience; 1993. 
25. Freedman LS. Tables of the number of patients required in clinical trials using the logrank test. Stat Med. 1982;1:121-9.

26. Gollnick HP. Oral retinoids-efficacy and toxicity in psoriasis. Br J Dermatol. 1996;135(Suppl 49):6-17.

27. Honda M, Yamashita T, Yamashita T, et al. Peretinoin, an acyclic retinoid, improves the hepatic gene signature of chronic hepatitis $\mathrm{C}$ following curative therapy of hepatocellular carcinoma. BMC Cancer. 2013;13:191.

28. Llovet JM, Di Bisceglie AM, Bruix J, et al. Design and endpoints of clinical trials in hepatocellular carcinoma. J Natl Cancer Inst. 2008;100:698-711.
29. Iwasaki Y, Ikeda H, Araki Y, et al. Limitation of combination therapy of interferon and ribavirin for older patients with chronic hepatitis C. Hepatology. 2006;43:54-63.

30. Nudo CG, Wong P, Hilzenrat N, et al. Elderly patients are at greater risk of cytopenia during antiviral therapy for hepatitis $\mathrm{C}$. Can J Gastroenterol. 2006;20:589-92. 